\title{
MEMBRANE SEPARATION IN FLOW INJECTION ANALYSIS Gas Diffusion
}

\author{
W. E. VAN DER LINDEN \\ Laboratory for Chemical Analysis, Department of Chemical Technology, Twente \\ University of Technology, P.O. Box 217, 7500 AE Enschede (The Netherlands)
}

(Received 20th January 1983)

\section{SUMMARY}

A general expression is derived for the membrane transport process in a flow-through unit as commonly used in flow injection systems. The validity of the formulae was tested for gas-diffusion membranes by using compounds with different volatilities such as ammonia, carbon dioxide and acetic acid. Several microporous hydrophobic membranes were tested. A new module design is proposed.

Membranes can be used in flow analysis systems in order to transfer certain compounds from a donor (sample) stream to an acceptor (detector) stream. This principle has been used for many years in continuous flow systems, with gas-diffusion and dialysis membranes. Baadenhuijsen and Seuren-Jacobs [1] seem to be the first who have exploited this concept in flow injection analysis (f.i.a.) in a procedure for the determination of carbon dioxide in plasma using a gas-permeable membrane. R ůžǐka and Hansen [2] refer to a similar procedure for the determination of ammonia, but details have not been published. As no quantitative description seems to have been presented of the membrane transport process in a flow-through unit and its dependence on the characteristic membrane parameters, it was decided to investigate this problem both from the theoretical and the practical point of view. The general equation derived was tested for some volatile compounds such as carbon dioxide, ammonia and acetic acid, with different types of gas-diffusion membranes.

\section{THEORY}

A mathematical relationship of the concentrations on both sides of the membrane as a function of time in dependence on the transfer coefficient and the flow rate can be derived starting from a tank-in-series model. In this model, the membrane separation unit (Fig. 1) is supposed to consist of a series of hypothetical units in each of which the solutions are homogeneous, i.e., ideally mixed (Fig. 2). If $\Delta V$ is taken as the volume on one side of the 


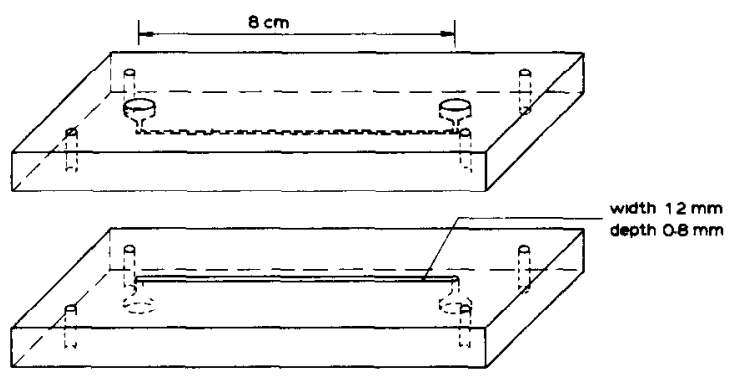

Fig. 1. Membrane separation module.

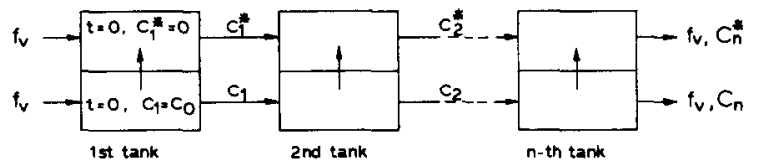

Fig. 2. Tanks-in-series model of membrane separation module.

membrane of such a hypothetical unit and it is assumed that the degree of transfer of the relevant compound is proportional to the concentration difference across the membrane, the following mass balances hold:

$\Delta V \mathrm{~d} C_{n} / \mathrm{d} t=-f_{v} C_{n}+f_{v} C_{n-1}-k\left(C_{n}-C_{n}^{*}\right)$

$\Delta V \mathrm{~d} C_{n}^{*} / \mathrm{d} t=-f_{v} C_{n}^{*}+f_{v} C_{n-1}^{*}+k\left(C_{n}-C_{n}^{*}\right)$

where the flow rate, $f_{v}$, is equal for both the donor and acceptor streams for simplicity reasons; $C_{n}$ is the concentration on the donor side, and $C_{n}^{*}$ is the concentration on the acceptor side, both for the $n$th separation unit (tank). Apart from the compound to be transferred, both streams are assumed to have basically the same composition. The transfer coefficient, $k$, is not specified here but will contain the parameters governing the different steps leading to the overall transfer: i.e., diffusion of the compound from the bulk to the solution/membrane interface, partition between donor solution and membrane phase, diffusion inside the membrane, partition between membrane and acceptor solution, and diffusion from the surface into the bulk of the acceptor phase.

Starting with the pulse-wise introduction of an amount of the relevant component corresponding to a concentration of $C_{0}$ in the first tank in the donor stream at $t=0$, Eqns. (1) and (2) can be solved (see Appendix A) leading to the general expressions:

$$
\begin{aligned}
& C_{n}=1 / 2 \frac{1}{(n-1) !}\left(f_{v} t / \Delta V\right)^{n-1} \exp \left[-f_{v} t / \Delta V\right]\{1+\exp [-2 k t / \Delta V]\} C_{0} \\
& C_{n}^{*}=1 / 2 \frac{1}{(n-1) !}\left(f_{v} t / \Delta V\right)^{n-1} \exp \left[-f_{v} t / \Delta V\right]\{1-\exp [-2 k t / \Delta V]\} C_{0}
\end{aligned}
$$


Already for $n>10$, the terms before the braces lead to approximately Gaussian peaks with a maximum at

$t_{\max }=(n-1) \Delta V / f_{v}$

and a variance of

$\sigma_{t}^{2}=(n-1)\left(\Delta V / f_{v}\right)^{2}=t_{\max }^{2} /(n-1)$

For larger values of $t$, the term within braces in Eqns. (3) and (4) will reach the value 1 and the compound will ultimately become equally distributed between the two streams.

In many practical applications, the component that permeates through the membrane will be chemically converted to a product, $P$ (e.g., $\mathrm{CO}_{2}+\mathrm{H}_{2} \mathrm{O} \rightleftharpoons$ $\mathrm{HCO}_{3}^{-}+\mathrm{H}_{3} \mathrm{O}^{+}$or $\mathrm{NH}_{3}+\mathrm{H}_{2} \mathrm{O} \rightleftharpoons \mathrm{NH}_{4}^{+} \perp \mathrm{OH}^{-}$). If the conditions in the acceptor stream are chosen in such a way that the conversion is complete and the stoichiometry of the reaction is $1: 1$, then the mass balance equations reduce to

$\Delta V \mathrm{~d} C_{n} / \mathrm{d} t=-f_{v} C_{n}+f_{v} C_{n-1}-k C_{n}$

$\Delta V \mathrm{~d} C_{p, n}^{*} / \mathrm{d} t=-f_{\nu} C_{p, n}^{*}+f_{v} C_{p, n-1}^{*}+k C_{n}$

where $C_{p, n}^{*}$ stands for the concentration of $P$ in the $n$th tank in the acceptor stream. According to the development presented in Appendix B, these equations lead to the general expressions:

$C_{n}=\frac{1}{(n-1) !}\left(f_{v} t / \Delta V\right)^{n-1} \exp \left[-f_{v} t / \Delta V\right] \exp [-k t / \Delta V] C_{0}$

$C_{p, n}^{*}=\frac{1}{(n-1) !}\left(f_{v} t / \Delta V\right)^{n-1} \exp \left[-f_{v} t / \Delta V\right]\{1-\exp [-k t / \Delta V]\} C_{0}$.

Starting from the same mass balances, Eqns. (3), (4), (9) and (10) can also be derived in an elegant way by means of Laplace transformation as shown by Reijn et al. [3] in a treatment of kinetics in f.i.a. In general, only the concentration $C^{*}$ leaving the membrane separation unit and transported to the detector, or detecting system, is of importance. Depending on the magnitude of the term $k t / \Delta V$, two extremes will be considered.

\section{Extreme cases}

Small kt/ $\Delta \mathrm{V}$. In the case of gas diffusion, this situation will occur, for instance, if the permeability is low or if the vapor pressure of the relevant component is relatively low, i.e., the compound is very readily soluble in the donor stream. Expansion of the exponential term within braces in Eqn. (10) leads to

$C_{p, n}^{*}=\frac{1}{(n-1) !}\left(f_{v} t / \Delta V\right)^{n-1} \exp \left[-f_{v} t / \Delta V\right]\left\{k t / \Delta V-1 / 2(k t / \Delta V)^{2}+\ldots\right\} C_{0}$ 
which approaches

$C_{p, n}^{*} \simeq \frac{1}{(n-1) !}\left(f_{v} t / \Delta V\right)^{n-1} \exp \left[-f_{v} t / \Delta V\right]\{k t / \Delta V\} C_{0}$

In an experimental set-up of constant geometry, the peak maximum is found at $t_{\mathrm{max}}=(n-1) \Delta V / f_{v}$, so $f_{v} t_{\max } / \Delta V$ is constant as long as the number of tanks can be considered to be constant. This situation is approximately warranted, e.g., in a single bead string reactor (SBSR) [4]. In that case

$\left(C_{p, n}^{*}\right)_{t_{\max }}=$ constant $\times(k t / \Delta V) C_{0}$

which means that the peak height is directly proportional to the residence time in the separation unit and inversely proportional to the flow rate.

Large $\mathrm{kt} / \Delta \mathrm{V}$. In this case, the exponential term within braces in Eqn. (10) approaches zero:

$C_{p, n}^{*}=\frac{1}{(n-1) !}\left(f_{v} t / \Delta V\right)^{n-1} \exp \left[-f_{v} t / \Delta V\right] C_{0}=$ constant $\times C_{0}$

and, again at a constant value of $n$, the peak height will become independent of the flow rate.

\section{EXPERIMENTAL}

\section{Chemicals and solutions}

All chemicals used were of analytical-reagent grade.

Indicator stock solutions. For the cresol red solution, $1 \mathrm{~g}$ of cresol red was dissolved in $20 \mathrm{ml}$ of $0.1 \mathrm{M} \mathrm{NaOH}$ and diluted to $1 \mathrm{l}$ with water. This indicator was used in combination with the carbonate buffer. For the phenolphthalein solution, $1 \mathrm{~g}$ of phenolphthalein was dissolved in $100 \mathrm{ml}$ of ethanol (96\%) and diluted to 11 with water. This indicator was used in combination with the ammonia buffer. For the bromocresol green solution, $2 \mathrm{~g}$ of bromocresol green was dissolved in $125 \mathrm{ml}$ of $0.1 \mathrm{M} \mathrm{NaOH}$ and diluted to $1 \mathrm{l}$ with water. This indicator was used in combination with the acetate buffer.

Buffer solutions. The $\mathrm{NaHCO}_{3} / \mathrm{Na}_{2} \mathrm{CO}_{3}, \mathrm{NH}_{4} \mathrm{Cl} / \mathrm{NH}_{3}$ and acetic acid/ sodium acetate buffers of the desired $\mathrm{pH}$ were prepared from $10^{-3} \mathrm{M}$ solutions of sodium carbonate, ammonium chloride or acetic acid, respectively, by adding hydrochloric acid or sodium hydroxide as appropriate.

Reagent solutions. Acceptor solutions were prepared by adding $10 \mathrm{ml}$ of the indicator stock solution to $1 \mathrm{l}$ of the buffer solution. The donor stream for the determination of ammonia was $0.1 \mathrm{M}$ sodium hydroxide, for carbon dioxide $0.1 \mathrm{M}$ sulphuric acid, and for acetic acid $1 \mathrm{M}$ sulphuric acid.

In the case of ammonia, Nessler's reagent was also used. This reagent was obtained from Merck, but a solution prepared by the procedure of Krug et al. [5] was also tested. Because the addition of Nessler's reagent to the acceptor stream caused clogging of the membrane pores, $0.1 \mathrm{M}$ sodium 
hydroxide was used as the primary acceptor solution, which was then mixed with the reagent in a mixing coil after the stream had passed the membrane unit, as shown schematically in Fig. 3. In this set-up, it was easy to interchange the outlets of the membrane unit so that the ammonia concentration in both the acceptor and the donor stream could be measured $\left(C\right.$ and $\left.C^{*}\right)$, thus allowing the calculation of an absolute value of the overall transfer of ammonia across the membrane.

\section{Equipment}

Membrane separation module. Three types were examined. The first was a laboratory-made module manufactured in perspex (see Fig. 1) with a groove of 8-cm length, $1.2-\mathrm{mm}$ width and $0.8-\mathrm{mm}$ depth; the groove was filled with polystyrene beads of approximately $0.6-\mathrm{mm}$ diameter. This module was used in all tests unless explicitly stated otherwise. The second was a laboratorymade module with a groove (10-cm long, $3.0-\mathrm{mm}$ wide and $0.2-\mathrm{mm}$ deep) with right-angled bends as shown in Fig. 4. The third was a commercial module (Tecator) with a groove $10 \mathrm{~cm}$ long, $2 \mathrm{~mm}$ wide and $0.2 \mathrm{~mm}$ deep.

The membranes tested are listed in Table 1.

Flow injection components. Absorbances were measured with a Metrohm E1009 spectrophotometer. The peristaltic pump was a Gilson Minipuls II. Polyethylene tubing (i.d. $0.8 \mathrm{~mm}$ ) was used. Because small pressure differences between the two channels in the separation module lead to deformation and possible perforation of the membranes, the tubes (transport lines and/or reaction/mixing coils) were not filled with beads, but coiled as tightly as possible in order to minimize dispersion. Injections were done with a Rheodyne teflon rotary valve type 50 provided with a pneumatic actuator. The injection volume was about $100 \mu \mathrm{l}$.

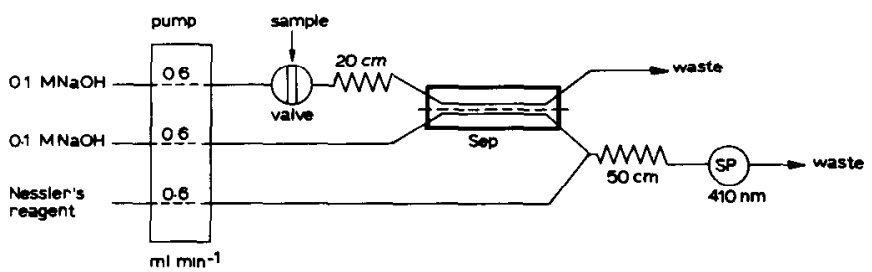

Fig. 3. Experimental set-up for the determination of ammonia with Nessler's reagent.

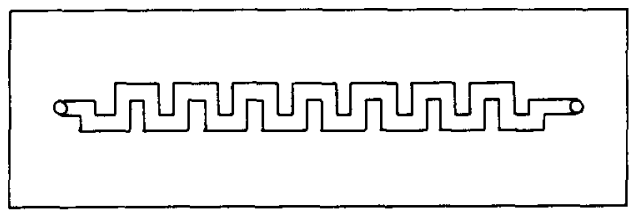

Fig. 4. Shape of the groove in the right-angled bends module. 


\section{TABLE 1}

Membranes examined

\begin{tabular}{lcc}
\hline & Pore diameter $(\mu \mathrm{m})$ & Thickness $(\mathrm{mm})$ \\
\hline PTFE (TBA) & $\leqslant 0.5$ & ca. $5 \times 10^{-2}$ \\
PTFE (Tecator) & $<0.5$ & ca. $3 \times 10^{-2}$ \\
Celgard $^{\mathrm{b}} 2400$ & 0.02 & $2.5 \times 10^{-2}$ \\
Celgard $^{\mathrm{b}} 2402$ & 0.02 & $5.0 \times 10^{-2}$ \\
Celgard $^{\mathrm{b}} 2500$ & 0.04 & $2.5 \times 10^{-2}$ \\
Celgard $^{\mathrm{b}} 3501$ & 0.04 & $2.5 \times 10^{-2}$ \\
\hline
\end{tabular}

${ }^{a}$ Both PTFE membranes are available as tape. Pore diameters are estimated from observations with an electron microscope. ${ }^{\mathrm{b}}$ Celgard is a microporous polypropylene film (Celanese Corporation, Charlotte, NC); 2400, 2402 and 2500 are hydrophobic films, whereas 3501 has a hydrophilic character [6].

\section{RESULTS AND DISCUSSION}

To obtain absolute values of the permeability of the membranes, the first experiments were done with ammonia and Nessler's reagent. Each experiment was duplicated, first with the outlet of the acceptor stream of the membrane module connected to the reagent stream, and then with the donor stream connected to the reagent stream (Fig. 3). The percentage transference across the membrane, expressed as $\left\{C^{*} /\left(C+C^{*}\right)\right\} \times 100$, was calculated for the injection of five different solutions containing $10^{-4}-5 \times 10^{-4} \mathrm{~mol} \mathrm{I}^{-1}$ ammonia. When the membranes are rather impermeable, the value of $C^{*}$ is so low that no reliable absorbance values could be obtained; in those cases, the concentration of the injected solution was increased five-fold. The standard deviation obtained from ten successive injections was found to be about $2 \%$. The results are summarized in Table 2 .

A good qualitative agreement can be observed between the permeability

\section{TABLE 2}

Percentage transference of ammonia across membranes in the concentration range $10^{-4}-$ $10^{-3} \mathrm{~mol} \mathrm{l}^{-1}$ at $20^{\circ} \mathrm{C}$

(Flow rate in both donor and acceptor streams was ca. $0.5 \mathrm{ml} \mathrm{min}{ }^{-1}$ )

\begin{tabular}{|c|c|c|c|c|c|c|}
\hline & \multirow{2}{*}{$\begin{array}{l}\text { PTFE } \\
\text { (TBA) }\end{array}$} & \multirow{2}{*}{$\begin{array}{l}\text { PTFE } \\
\text { (Tecator) }\end{array}$} & \multicolumn{4}{|c|}{ Celgard $^{a}$} \\
\hline & & & 2400 & 2402 & 2500 & 3501 \\
\hline Home-made module ${ }^{b}$ & 9.2 & - & $\begin{array}{l}2.40 \\
2.36\end{array}$ & $\begin{array}{l}1.03 \\
0.95\end{array}$ & $\begin{array}{l}7.2 \\
9.6\end{array}$ & $\begin{array}{l}1.38 \\
0.92\end{array}$ \\
\hline Tecator module & 20.2 & 24.6 & - & - & - & - \\
\hline
\end{tabular}

a Each value is the result of a complete set of measurements of at least five different concentrations. ${ }^{b}$ As shown in Fig. 1. 
and the characteristic membrane parameters such as pore size and thickness. Between Celgard 2400 and 2402 a two-fold decrease in transference is to be expected. The slightly larger decrease might be due to the fact that the 2402 film is not homogeneous but consists of two 2400 films on top of each other introducing extra boundary effects. The three- to four-fold increase in transference for the 2500 film compared to the 2400 film is in good agreement with the four-fold increase expected from doubling the pore diameter. The PTFE (TBA) tape has about the same permeability for ammonia as the 2500 Celgard film although it seems to have larger pores. Electron microscopy revealed, however, that the membrane is rather inhomogeneous and that the number of these larger pores is limited. The PTFE tape supplied by Tecator seems to be a little better in this respect. Both PTFE tapes are easily stretched, which might cause a change of pore shape and even the number of pores. This may explain why each new membrane of the same tape yields slightly different results. The difference between the laboratory-made and the Tecator module corresponds approximately to the 2.5-3 times increase in the contact surface of solutions and membrane. Celgard 3501 tended to swell on contact with water, which led to an undefined effective film thickness. Therefore, and because its permeability was not favourable compared to the other membranes, this film was not used in further experiments.

The relatively low permeability for ammonia suggests that $2 k t / \Delta V$ is small. Equation (4) can then be reduced to an expression similar to Eqn. (12) and the absorbance at $t_{\max }$ plotted as a function of the inverse flow rate must yield a straight line through the origin. This was actually found, as can be seen in Fig. 5. At the same time, $C_{n}$ should remain constant according to Eqn. (4). It has to be kept in mind, however, that in using Eqns. (3) and (4) it is assumed that no extra band broadening occurs in the tubes to the membrane module and from this module to the detector. This might be the reason why $C_{n}$ is not exactly constant for the various inverse flow rates.

Because in practice it is much easier to work with an acid-base indicating system instead of Nessler's reagent, the experiments were also done with a phenolphthalein-ammonium buffer system as the acceptor solution. The best choice of $\mathrm{pH}$ depends on the $\mathrm{p} K_{\mathrm{a}}$ value of the buffer substance, the $\mathrm{p} K_{\mathrm{a}}$ value of the indicator and the concentration range to be measured. Theoretical considerations about such a choice will be presented in a separate paper. At this stage, it was found experimentally that for $\mathrm{pH} 9.0$ good linear calibration curves were obtained (Fig. 6). Although measured with a completely new set of membranes, the same order of permeability is again observed: the PTFE membranes show the best permeability, the Celgard 2500 is almost as good, whereas the Celgard films 2400 and 2402 are permeable only to a limited extent.

For microporous PTFE and polypropylene membranes, the degree of transfer depends mainly on the volatility of the compound, as has been noted by Kobos et al. [7]. Accordingly, it is to be expected that carbon dioxide will show a much greater permeability than will ammonia; the solu- 

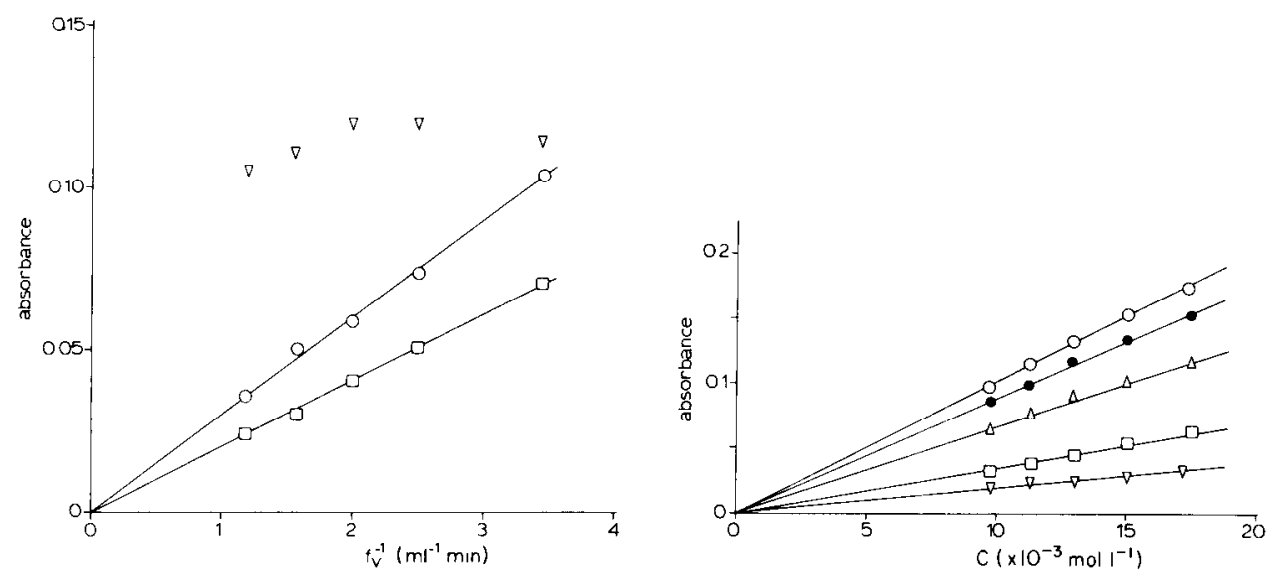

Fig. 5. Absorbance at peak maximum versus the inverse flow rate of the acceptor/donor stream, for the Celgard 2500 membrane at $20^{\circ} \mathrm{C}$. Concentration injected: (0) $1.5 \times 10^{-3}$ mol l-1; (ㅁ) $1.2 \times 10^{-3} \mathrm{~mol} \mathrm{l}^{-1}$; ( $\left.\nabla\right) 2.4 \times 10^{-4} \mathrm{~mol} \mathrm{l} \mathrm{l}^{-1}$. (०, 口) Absorbance measured in the acceptor stream, $(\nabla)$ absorbance measured in the donor stream.

Fig. 6. Absorbance at peak maximum vs. in jected concentration of ammonia (as ammonium chloride). Volume injected, ca. $100 \mu \mathrm{l}$; flow rate, $0.5 \mathrm{ml} \mathrm{min}{ }^{-1}$; buffer solution, $10^{-3} \mathrm{M}$ $\left(\mathrm{NH}_{4} \mathrm{Cl}+\mathrm{NH}_{3}\right)+$ phenolphthalein, pH 9.0. Membrane: (०) PTFE (TBA); (•) PTFE (Tecator); ( $(\square)$ Celgard 2400; $(\nabla)$ Celgard 2402; $(\Delta)$ Celgard 2500.
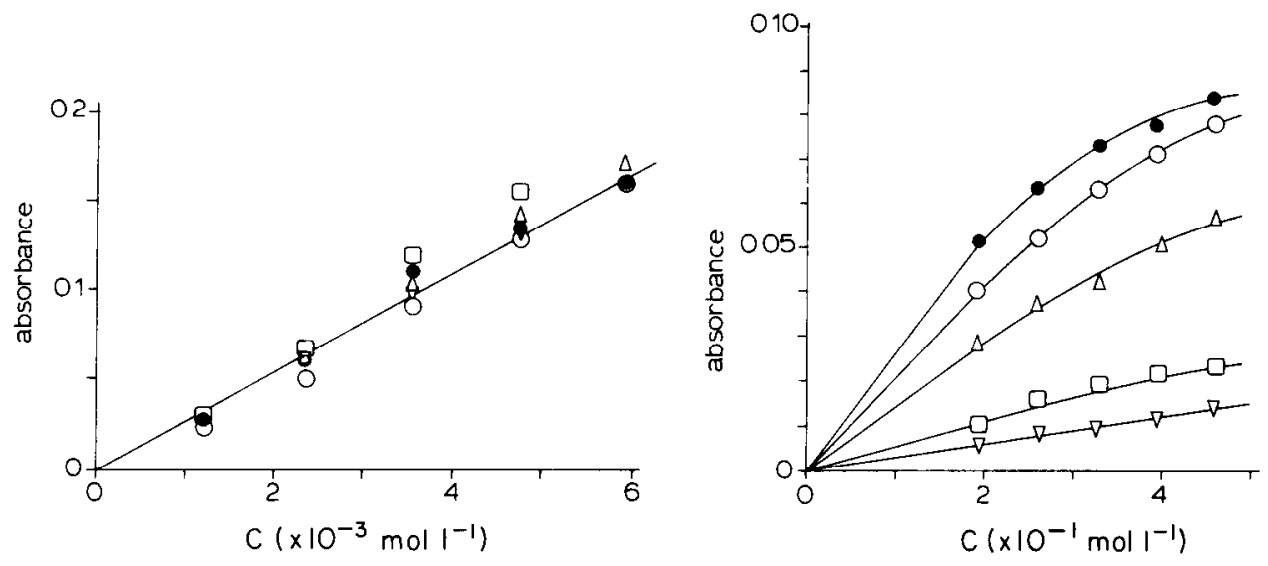

Fig. 7. Absorbance at peak maximum vs. injected concentration of carbon dioxide (as

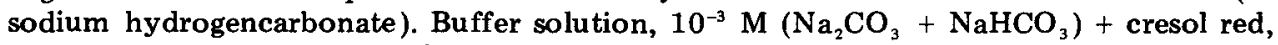
pH 8.8 ; flow rate $0.6 \mathrm{ml} \mathrm{min}{ }^{-1}$; other details as for Fig. 6 .

Fig. 8. Absorbance at peak maximum vs. injected concentration of acetate. Buffer solu-

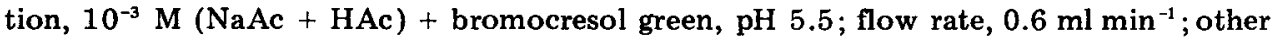
details as for Fig. 6. 
bility of ammonia at $20^{\circ} \mathrm{C}$ is $0.515 \mathrm{~g} \mathrm{~g}^{-1}$ of water [8] whereas the solubility of carbon dioxide at $20^{\circ} \mathrm{C}$ is $0.00172 \mathrm{~g} \mathrm{~g}^{-1}$ of water [9]. In the case of carbon dioxide, $k t / \Delta V$ will become large for all the membranes under investigation and Eqn. (13) will apply, which means that the calibration curves for all membranes must coincide. This was found experimentally, as depicted in Fig. 7.

Acetic acid was used as another example. As acetic acid is infinitely soluble in water, it was expected that transference across the membranes would be even more limited than for ammonia. This was actually observed; much larger concentrations had to be injected in order to achieve any response. Probably because of the use of such high concentrations, the calibration curves are not exactly linear. As for ammonia, the slopes of the calibration curves for the various membranes were different (Fig. 8).

Especially for ammonia and acetic acid the volatility, and hence the transference across the membrane, can be increased by raising the temperature. For both ammonia and acetic acid, a markedly enhanced signal was observed by raising the temperature from $20^{\circ} \mathrm{C}$ to $60^{\circ} \mathrm{C}$. For ammonia, this increase was found to be approximately inversely proportional to the solubility as shown in Fig. 9. For carbon dioxide the temperature-dependence was less pronounced. It must be stated, however, that the results of these temperature experiments must be considered with great caution because changing the temperature can shift not only the value of the molar absorptivity but also all the equilibria.

In conclusion, it has been proven that the general equations derived are essentially correct. To obtain maximum sensitivity in the case of less volatile compounds, it is advisable to use microporous PTFE membranes or the microporous polypropylene film commercially available as Celgard 2500 . Because the latter is less prone to electrostatic charging, it is easier to handle, and it is available as sheets from which membranes of any shape or size can be cut.

For less volatile compounds it is advisable to increase the proportionality factor $(k)$ for the mass transfer across the membrane by increasing the active surface area. In order to accomplish this and to maintain the condition of

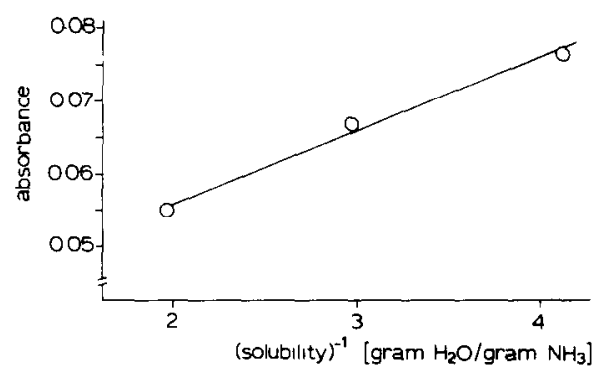

Fig. 9. Absorbance at peak maximum vs. inverse solubility of ammonia in water at different temperatures. 
ideal mixing, a membrane module with right-angle bends was constructed (Fig. 4). Preliminary experiments showed that the two- to three-fold increase in membrane surface area leads indeed to a corresponding increase in signal, whereas the peak width hardly changes. With a flow rate of $1 \mathrm{ml} \mathrm{min}{ }^{-1}$, a sample rate of 70 samples $\mathrm{h}^{-1}$ is feasible without any carryover.

Although reproducible measurements can be made in a fixed set-up with one membrane, it must be kept in mind that regular calibration is necessary and certainly is a prerequisite when the membrane is changed, even when it is taken from the same tape or film.

The author expresses his gratitude to $R$. Wasser and $M$. Scheltinga for their experimental help, to Dr. Ir. M. Bos for his critical comments on the manuscript, to Mrs. B. Verbeeten-van Hettema for preparing the manuscript and to R. H. Arends for making the drawings. The Celgard films were kindly provided by Celanese Corporation (USA), and a membrane module and tape by Tecator (Sweden).

\section{APPENDIX A}

Mass balances for the first tank are

$$
\mathrm{d} C_{1} / \mathrm{d} t=-C_{1} f_{v} / \Delta V-\left(C_{1}-C_{1}^{*}\right) k / \Delta V
$$$$
\mathrm{d} C_{1}^{*} / \mathrm{d} t=-C_{1}^{*} f_{v} / \Delta V+\left(C_{1}-C_{1}^{*}\right) k / \Delta V
$$

Combination of (A1) and (A2) yields $\mathrm{d}\left(C_{1}+C_{1}^{*}\right) / \mathrm{d} t=\left(C_{1}+C_{1}^{*}\right) f_{v} / \Delta V$, and integration leads to $C_{1}+C_{1}^{*}=$ const. $\exp \left[-f_{v} t / \Delta V\right]$. For the starting conditions $t=0, C_{1}=C_{0}, C_{1}^{*}=0$, the result obtained is $C_{1}+C_{1}^{*}=C_{0} \exp \left[-f_{v} t \Delta V\right]$. Introduction of this equation in Eqns. (A1) and (A2) leads to

$$
\begin{aligned}
& \mathrm{d} C_{1} / \mathrm{d} t=-C_{1} f_{v} / \Delta V-\left(C_{1}-C_{0} \exp \left[-f_{v} t / \Delta V\right]+C_{1}\right) k / \Delta V \rightarrow \mathrm{d} C_{1} / \mathrm{d} t+C_{1}\left(f_{v}+2 k\right) / \Delta V \\
& \quad=C_{0} \exp \left[-f_{v} t / \Delta V\right]
\end{aligned}
$$

This Leibniz-type linear differential equation has the general solution

$$
C_{1}=1 / 2 C_{0} \exp \left[-f_{v} t / \Delta V\right]+a \exp \left[-\left(f_{v}+2 k\right) t / \Delta V\right]+b
$$

where $a$ and $b$ are constants.

For $t \rightarrow \infty, C_{1} \rightarrow 0$ so $b=0$; for $t=0, C_{1}=1 / 2 C_{0}+a=C_{0}$ so $a=1 / 2 C_{0}$. This leads to the expression

$$
C_{1}=1 / 2 C_{0} \exp \left[-f_{v} t / \Delta V\right]\{1+\exp [-2 k t / \Delta V]\}
$$

Similarly,

$$
C_{1}^{*}=1 / 2 C_{0} \exp \left[-f_{v} t / \Delta V\right]\{1-\exp [-2 k t / \Delta V]\}
$$

For the second tank the mass balances yield

$$
\begin{aligned}
& \mathrm{d} C_{2} / \mathrm{d} t=+C_{1} f_{v} / \Delta V-C_{2} f_{v} / \Delta V-\left(C_{2}-C_{2}^{*}\right) k / \Delta V \\
& \mathrm{~d} C_{2}^{*} / \mathrm{d} t=+C_{1}^{*} f_{v} / \Delta V-C_{2}^{*} f_{v} / \Delta V+\left(C_{2}-C_{2}^{*}\right) k / \Delta V
\end{aligned}
$$

Thus

$$
d\left(C_{2}+C_{2}^{*}\right) / \mathrm{d} t=\left(C_{1}+C_{1}^{*}\right) f_{v} / \Delta V-\left(C_{2}+C_{2}^{*}\right) f_{v} / \Delta V
$$


which again is a linear differential equation of the Leibniz-type. With the boundary condition $t=0 \rightarrow C_{2}+C_{2}^{*}=0$, this leads to

$C_{2}+C_{2}^{*}=-C_{0} t f_{v} / \Delta V \exp \left[-f_{v} t / \Delta V\right]$

and after substitution in Eqns. (A7) and (A9)

$C_{2}=-C_{1} t f_{v} / \Delta V=1 / 2 C_{0}\left(f_{v} t / \Delta V\right) \exp \left[-f_{v} t / \Delta V\right]\{1+\exp [-2 k t / \Delta V]\}$

$C_{2}^{*}=1 / 2 C_{0}\left(f_{v} t / \Delta V\right) \exp \left[-f_{v} t / \Delta V\right]\{1-\exp [-2 k t / \Delta V]\}$

Continuation through the subsequent tanks leads eventually for the $n$th tank to Eqns. (3) and (4) in the main text.

\section{APPENDIX B}

Mass balances for the first tank are

$\mathrm{d} C_{1} / \mathrm{d} t=-\left(f_{v} / \Delta V\right) C_{1}-k C_{1}$

$\mathrm{d} C_{p, 1}^{*} / \mathrm{d} t=-\left(f_{v} / \Delta V\right) C_{p, 1}^{*}+k C_{1}$

where $C_{D, 1}^{*}$ is the concentration of the product, $P$, resulting from a $1: 1$ stoichiometric reaction. Equation (B2) only holds for a 1:1 stoichiometry of the reaction. Equation (B1) leads in a straightforward way to

$C_{1}=C_{0} \exp \left[-t\left(f_{v}+k\right) / \Delta V\right]$

Substitution in Eqn. (B2) yields

$C_{p, 1}^{*}=C_{0} \exp \left[-f_{v} t / \Delta V\right]\{1-\exp [-k t / \Delta V]\}$

For the $n$th tank, continuation of the derivation leads to Eqns. (9) and (10) in the main text.

\section{REFERENCES}

1 H. Baadenhuijsen and H. E. H. Seuren-Jacobs, Clin. Chem., 25 (1979) 443.

2 J. Růžicka and E. H. Hansen, Flow Injection Analysis, Wiley, New York, 1981.

3 J. M. Reijn, W. E. van der Linden and H. Poppe, submitted for publication.

4 J. M. Reijn, W. E. van der Linden and H. Poppe, Anal. Chim. Acta, 126 (1981) 1.

5 F. J. Krug, J. R\&žicka and E. H. Hansen, Analyst, 104 (1979) 47.

6 H. S. Bierenbaum, R. B. Isaacson, M. L. Druin and S. G. Plovan, Ind. Eng. Chem., Prod. Res. Develop., 13 (1974) 2.

7 R. K. Kobos, S. J. Parks and M. E. Meyerhoff, Anal. Chem., 54 (1982) 1976.

8 W. Braker, Matheson Gas Data Book, Matheson Gas Prod., Milwaukee, 1971.

9 L. Medard (Ed.), L'Air Liquide, Gas Encyclopedia, Elsevier, 1976. 